\title{
Folic acid receptor-targeted human serum albumin nanoparticle formulation of cabazitaxel for tumor therapy
}

This article was published in the following Dove Press journal: International Journal of Nanomedicine

\section{Yating Sun' \\ Yarong Zhao' \\ Shanshan Teng' \\ Fei Hao' \\ Huan Zhang' \\ Fanchao Meng' \\ Xiuting Zhao' \\ Xiaolong Zheng' \\ Ye $\mathrm{Bi}^{\prime}$ \\ Yicheng Yao ${ }^{2}$ \\ Robert J Lee ${ }^{1,3}$ \\ Lesheng Teng'}

'School of Life Sciences, Jilin University, Changchun, Jilin, China;

${ }^{2}$ Acalanes High School, Lafayette, CA, USA; ${ }^{3}$ Division of Pharmaceutics, College of Pharmacy, The Ohio State University, Columbus, OH, USA
Correspondence: Lesheng Teng School of Life Sciences, Jilin University, 2699 Qianjin Avenue, Changchun, Jilin, China

Tel/fax+8643I85I5 5320

Email tenglesheng@jlu.edu.cn
Background: We previously developed cabazitaxel (CTX)-loaded human serum albumin nanoparticles (NPS-CTX) via a self-assembly method, and these NPs showed efficacy in prostate cancer therapy. Many studies have shown that the levels of folic acid (FA) receptor on the surface of various tumor cells are high. Therefore, FA-modified NPs-CTX may have enhanced antitumor effects compared with unmodified NPs-CTX.

Methods: NPs-CTX were first prepared via self-assembly, and FA was conjugated on the surface of NPs-CTX through the - $\mathrm{NH}_{2}$ groups of the NPs to produce FA-NPs-CTX. The FA-NPs-CTX were evaluated in tumor cells with high FA receptor (FR) expression in vitro and in vivo.

Results: Both NPs-CTX and FA-NPs-CTX exhibited good stability and morphology. Drug release from the NPs was not affected by FA conjugation. Compared with CTX dissolved in a mixture of Tween 80 and $13 \%$ ethanol $(\mathrm{w} / \mathrm{w})$ at a ratio of 1:4 (v/v) (Tween-CTX), the two nanoformulations had lower lytic activity against normal red blood cells. However, FA-NPs-CTX showed greater inhibition of tumor cells with overexpressed FR, compared with NPs-CTX, in the cytotoxicity experiments. Moreover, the cellular uptake of FA-NPs-CTX was enhanced through FR-mediated endocytosis in HeLa cells in vitro and HeLa xenograft tumors in vivo. Although Tween-CTX exhibited tumor growth inhibition similar to FA-NPs-CTX in vivo, this inhibition also caused adverse side effects; the median lethal dose (LD50) of Tween-CTX to mice was $5.68 \mathrm{mg} / \mathrm{kg}$, while FA-NPs-CTX-treated mice survived at doses exceeding $400 \mathrm{mg} / \mathrm{kg}$.

Conclusion: The results showed that FA-NPs-CTX caused inhibition of tumor growth in a manner similar to that of Tween-CTX; however, the safety and tolerability of CTX were greatly improved by FA conjugation compared with those of Tween-CTX. In summary, FA-NPs-CTX have great potential in CTX delivery, and this formulation is a promising candidate for the treatment of cancers with high FR levels.

Keywords: folic acid, human serum albumin, nanoparticles, cabazitaxel, folic acid receptor

\section{Introduction}

Cabazitaxel (CTX) belongs to the taxane group of anticancer agents and is a second-line drug for prostate cancer, developed by Sanofi-Aventis (Bridgewater, NJ, USA). CTX is used to treat taxane-resistant tumors and has shown superior efficacy to docetaxel. ${ }^{1,2}$ Unlike docetaxel, CTX is not pumped out by P-glycoprotein (P-gp) after endocytosis by cells due to its low substrate affinity for P-gp. ${ }^{3,4}$ CTX was approved by the US Food and Drug Administration (FDA) to treat prostate cancer in 2010. However, its clinical application has been limited due to low solubility. ${ }^{5}$ As the only commercialized CTX formulation, Jevtana (Sanofi-aventis, Paris, France) often causes serious side effects and allergic reactions such as hypotension and generalized rash/erythema due to the 
use of polysorbate 80 (Tween 80 ) and dehydrated ethanol as cosolvents. ${ }^{6,7}$ Moreover, this type of CTX formulation is rapidly cleared from the body and may not accumulate at tumor sites. Therefore, the development of a nanoformulation that can enhance the solubility and tumor bioavailability of CTX while reducing its side effects is desirable.

Nanovehicles (liposomes, micelles, and nanoparticles [NPs]) have attracted increasing attention in recent years. ${ }^{8}$ With the FDA approval of Abraxane ${ }^{\circledR}$ (Abraxis BioScience, Los Angeles, CA, USA), ${ }^{9}$ human serum albumin (HSA) NPs have been extensively investigated. ${ }^{10} \mathrm{HSA}$ is a major component in serum and has the advantages of inherent biocompatibility..$^{8,11,12}$ NPs can increase the solubility of hydrophobic drugs in aqueous solution ${ }^{13}$ and can passively accumulate in tumor tissue through the enhanced permeability and retention (EPR) effect. ${ }^{14}$ Additionally, many targeting ligands (transferrin, folic acid [FA], human epidermal growth factor receptor 2 [HER2], and so on) can be conjugated to the surface of NPs for tumor cell targeting. Normally, the targeting ligands are aimed toward receptors that are overexpressed on tumor cells, such as FR. FR is a glycoprotein that is overexpressed on tumor cells (breast and ovarian cancers). ${ }^{15,16} \mathrm{FA}$ can bind to FR and promote the uptake of FA-targeted NPs by receptor-mediated endocytosis (Figure 1). ${ }^{17-19}$
In previous studies, ${ }^{20,21}$ we prepared HSA-loaded CTX NPs (NPs-CTX) by a self-assembly method and used them for prostate tumor therapy in a PC-3 xenograft model. The NPs showed good efficacy in inhibiting PC-3 growth and exhibited a good safety profile. To further improve the efficiency of CTX delivery, FR-targeted NPs-CTX (FA-NPs-CTX) were prepared and applied to FR-overexpressing HeLa cells. ${ }^{16}$ The properties of NPs-CTX and FA-NPs-CTX, including particle size, zeta potential, stability, drug loading efficiency, drug release, and tumor growth inhibition, were investigated. Additionally, the cellular uptake of FA-NPs-CTX was evaluated - in comparison with NPs-CTX - by flow cytometry and confocal laser scanning microscopy (CLSM). Moreover, 1,1'-dioctadecyl-3,3,3',3'-tetramethyl indotricarbocyanine (DiR)-loaded FA-NPs-DiR and NPs-DiR were used to investigate tumor accumulation parameters with an IVIS ${ }^{\circledR}$ imaging system. The therapeutic efficacy of FANPs-CTX against HeLa tumor xenograft-bearing mice was evaluated in comparison with NPs-CTX in vivo.

\section{Materials and methods Materials}

CTX was purchased from Yew Biological Technology Co, Ltd (Wuxi, China). HSA was from Octapharma (Vienna,

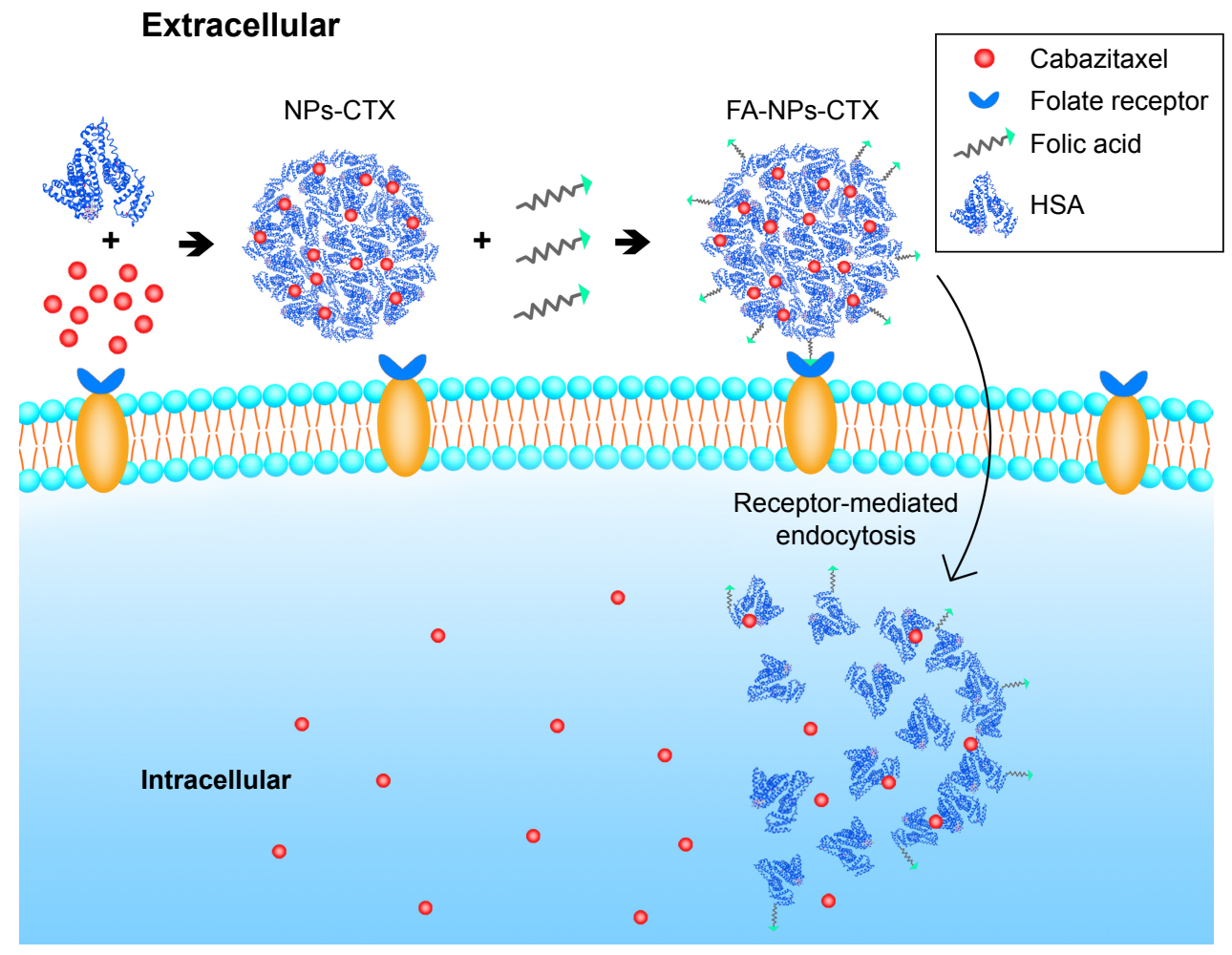

Figure I Schematic representation of the FR-mediated delivery of FA-NPs-CTX into cells.

Abbreviations: CTX, cabazitaxel; FA, folic acid; FA-NPs-CTX, FR-targeted cabazitaxel-loaded nanoparticles; FR, folic acid receptor; HSA, human serum albumin; NPs-CTX, cabazitaxel-loaded nanoparticles. 
Austria). FA and $N$-hydroxysuccinimide (NHS) were obtained from Yuan Ye Biotechnology Co, Ltd (Shanghai, China). $N, N^{\prime}$-Dicyclohexylcarbodiimide (DCC), fluorescein isothiocyanate (FITC), and DAPI were purchased from Sigma-Aldrich (St Louis, MO, USA). DiR iodide was purchased from Thermo Fisher Scientific (Waltham, MA, USA). All other reagents were of analytical grade.

\section{Cells and animals}

HeLa and A549 cells were obtained from ATCC and cultured in high-glucose DMEM supplemented with $10 \% \mathrm{FBS}$, $100 \mu \mathrm{g} / \mathrm{mL}$ of streptomycin, and $100 \mathrm{IU} / \mathrm{mL}$ penicillin $\left(37^{\circ} \mathrm{C}\right.$, $5 \% \mathrm{CO}_{2}$ ).

Female BALB/c nude mice (8-9 weeks old, 18-22 g) and $\mathrm{BALB} / \mathrm{c}$ mice (male and female, 18-22 g) were purchased from Beijing Vital River Laboratory Animal Technology Co, Ltd (Beijing, China). Mice were managed and maintained according to the requirements of the barrier environment facilities. Animals had free access to food and water. All animal experiments were approved by the institution animal ethics committee (Jilin University) (number 201709006). All experimental procedures were conducted in accordance with the Guidelines on Humane Treatment of Laboratory Animals (published in 2009) and procedures for the care and use of laboratory animals.

\section{Preparation of NPs-CTX and NPs-DiR}

NPs-CTX were prepared as described previously. ${ }^{20}$ Briefly, $3 \mathrm{~mL}$ of $12 \%$ sodium chloride solution was added to $2 \mathrm{~mL}$ of HSA. This solution was mixed with $6 \mathrm{~mL}$ of $10 \mathrm{mg} / \mathrm{mL} \mathrm{CTX}$ in ethanol and injected into rapidly stirred deionized water at $65^{\circ} \mathrm{C}$. Next, the solution was quickly cooled to $2^{\circ} \mathrm{C}-8^{\circ} \mathrm{C}$, and NPs-CTX were formed. The obtained NPs-CTX were concentrated by hollow fiber ultrafiltration to remove ethanol and unencapsulated CTX. Finally, the NPs-CTX were freezedried. NPs-DiR were prepared using the same protocol as for NPs-CTX, except that the ethanol solution of CTX was replaced with an ethanol solution of DiR during the preparation process.

\section{Preparation of FA-NPs-CTX, FA-NPs-DiR, and FITC-labeled NPs}

FA-NPs-CTX were prepared through reaction between FANHS and NPs-CTX. FA-NHS was synthesized as described previously. ${ }^{22}$ Briefly, $300 \mathrm{mg}$ FA was dissolved in $6 \mathrm{~mL}$ dimethyl sulfoxide (DMSO). Then, $150 \mu \mathrm{L}$ triethylamine was added, and $282 \mathrm{mg}$ DCC and $156 \mathrm{mg}$ NHS were subsequently added. The mixture was stirred overnight at room temperature. The insoluble by-product, dicyclohexylurea (DCU), was removed by filtration, and FA-NHS was precipitated by pouring the filtrate into an ice-cold solution of anhydrous ether containing $30 \%$ acetone. The precipitate was centrifuged at $12,857 \times g$ for 10 minutes at $4^{\circ} \mathrm{C}$ and washed with anhydrous ether. FA-NHS was obtained after suction filtration and vacuum-drying.

A small quantity of FA-NHS (10 mg) was dissolved in $2 \mathrm{~mL}$ of carbonate/bicarbonate buffer solution $(0.2 \mathrm{M}$, $\mathrm{pH}$ 10), and the freeze-dried NPs-CTX were dissolved in the same buffer solution. FA-NHS was added dropwise into NPs-CTX, and the solution was gently stirred for 2 hours. The FA-NPs-CTX solution was obtained by centrifugal ultrafiltration (50 kDa, 3,214× $g, 10$ minutes) and was washed with deionized water. The product was then freeze-dried. FA-NPs-DiR were prepared with the same protocol as for FA-NPs-CTX.

FITC-labeled HSA was synthesized as described previously. HSA was first diluted in a $\mathrm{Na}_{2} \mathrm{CO}_{3} / \mathrm{NaHCO}_{3}$ buffer solution ( $25 \mathrm{mM}, \mathrm{pH}=9.8$ ) to a concentration of $5 \mathrm{mg} / \mathrm{mL}$. Then, FITC was added to the HSA solution at a final concentration of $0.1 \mathrm{mg} / \mathrm{mL}$, and the solution was incubated overnight at room temperature. The final product was obtained by centrifuging the mixture solution in ultrafiltration tubes with a molecular weight cutoff of $50 \mathrm{kDa}$ at $12,857 \times g$ for 10 minutes. The product was washed with the same $\mathrm{Na}_{2} \mathrm{CO}_{3} / \mathrm{NaHCO}_{3}$ buffer and centrifuged twice to obtain FITC-labeled HSA (FITC-HSA). FITC-NPs-CTX and FA-FITC-NPs-CTX were prepared using the same procedure as the nonlabeled NPs.

\section{Characterization and evaluation of NPs}

The particle size and zeta potential of NPs-CTX and FANPs-CTX were measured on a Zetasizer NP size analyzer (Nano-ZS ZEN3600; Malvern Instruments, Malvern, UK) after dissolution in deionized water. The morphologies of the two NPs were determined by scanning electron microscopy (SEM; JSM-6700F; JEOL, Tokyo, Japan). One drop of NPs diluted in deionized water was added to the surface of silicon wafer and air-dried. The samples were then sputter-coated with platinum and imaged with SEM.

The quantity of FA conjugated to FA-NPs-CTX was measured using a spectrophotometric method as described previously. ${ }^{22}$ Briefly, FA-NPs-CTX were digested using trypsin in phosphate buffer $(\mathrm{pH}=7.4)$ for 4 hours, and the digestion product was scanned in the range of 200-400 nm with a UV spectrophotometer (UV2401; Shimadzu Corporation, Kyoto, Japan); the tryptic hydrolysis of NPs-CTX 
was used as a blank control. The absorbance of the digested product at $352 \mathrm{~nm}$ was used to calculate the quantity of FA conjugated to FA-CTX-NPs using a calibration curve prepared with a standard solution of FA.

The drug encapsulation efficiency (EE) and encapsulated content of drug (EC) of NPs were measured by HPLC and calculated using the following equations. Briefly, for calculating the EE of NPs-CTX, the concentrations of CTX loaded in the NPs-CTX and the unencapsulated CTX present in the filtrate were detected, respectively, by injecting $20 \mu \mathrm{L}$ samples into the HPLC setup (LC-20AD; Shimadzu Corporation) with a HC-C18 column $(4.6 \times 150 \mathrm{~mm}$; Agilent Technology Co., Ltd., Beijing, China). The detection wavelength was $230 \mathrm{~nm}$, and the mobile phase was a mixture of acetonitrile and ultrapure water at a mixing ratio 60:40. For EC, freeze-dried NPs-CTX and FA-NPs-CTX were dissolved in acetonitrile and sonicated to release the CTX, and then the diluted CTX was injected into the HPLC setup.

$$
\begin{aligned}
\mathrm{EE}= & \frac{\text { Weight of CTX in NPs }}{(\text { Weight of CTX in NPs }} \times 100 \% \\
& + \text { Weight of CTX filtrate) } \\
\mathrm{EC} & =\frac{\text { Weight of CTX in NPs }}{\text { Weight of NPs }} \times 100 \%
\end{aligned}
$$

\section{Stability evaluation}

To study the stability of NPs in vitro, freeze-dried FA-NPsCTX and NPs-CTX were dissolved in normal saline (NS) and $10 \%$ FBS separately. Then, the NPs were placed at $25^{\circ} \mathrm{C}$ and $37^{\circ} \mathrm{C}$, respectively, and aliquots of NPs were removed at fixed time points for measuring particle size. The colloidal stability of NPs was reflected by the changes of the NPs' particle size.

\section{In vitro release test}

Drug release from Tween-CTX, NPs-CTX, and FA-NPsCTX was determined in PBS $(\mathrm{pH}=7.4)$ containing $0.1 \%$ $(\mathrm{v} / \mathrm{v})$ Tween 80 at $37^{\circ} \mathrm{C}$. Appropriate aliquots $(2 \mathrm{~mL}$ each) of Tween-CTX (CTX dissolved in a mixture of Tween 80 and $13 \%$ ethanol $(\mathrm{w} / \mathrm{w})$ at a ratio of $1: 4(\mathrm{v} / \mathrm{v}))$, NPs-CTX, and FA-NPs-CTX were placed in a dialysis bag (with a molecular weight cutoff of $10 \mathrm{kDa}$ ) separately. These dialysis bags were completely immersed in $80 \mathrm{~mL}$ release medium with magnetic stirring at $200 \mathrm{rpm}$. Aliquots of samples were withdrawn at predetermined time intervals and replaced with the same volumes of release medium. The concentration of CTX was determined by HPLC as described earlier.

\section{In vitro cytotoxicity of CTX-loaded NPs}

The cytotoxicity of FA-NPs-CTX was determined with the MTT assay. Human cervical cancer HeLa cells (with FR overexpression) and human non-small-cell lung cancer A549 cells (FR negative) were used to evaluate the inhibitory effect of FA-NPs-CTX. NPs-CTX and free CTX (dissolved in ethanol) were used as controls. Briefly, cells were seeded at a density of 5,000-8,000 cells per well in a 96-well plate and incubated for 24 hours to adhere. Free CTX, NPs-CTX, and FA-NPs-CTX solutions, which contained CTX concentrations of 20,60 , or $150 \mu \mathrm{g} / \mathrm{mL}$, were added to the cells, which were cultured for 24,48 , or 72 hours. Then, $10 \mu \mathrm{L}$ of MTT ( $5 \mathrm{mg} / \mathrm{mL}$ ) was added to each well, and the cells were incubated at $37^{\circ} \mathrm{C}$ for 4 hours. The supernatant was removed, DMSO was added (100 $\mu \mathrm{L} /$ per well), and absorbance at $490 \mathrm{~nm}$ was measured.

\section{Cellular uptake assessment}

Flow cytometry was used to quantify the cellular uptake of labeled NPs. HeLa and A549 cells were inoculated into sixwell plates at a density of $2 \times 10^{5}$ cells per well and cultured overnight. Then, FITC-labeled NPs (FITC-NPs-CTX and FA-FITC-NPs-CTX) with equal protein concentrations were separately added into the wells and incubated with cells for 1,2 , or 4 hours. To investigate the FR targeting of FA-NPsCTX, free FA ( $5 \mathrm{mM})$ was added to the medium 1 hour before drug administration for competitive FR blockade. To quantify the fluorescence intensity in cells, cells were trypsinized and washed with ice-cold PBS three times, centrifuged at $82 \times g$, resuspended in $4 \%$ paraformaldehyde, and examined by flow cytometry (Coulter Epics XL, Beckman Coulter, Brea, CA, USA).

LCSM was further performed to visualize the uptake of FITC-NPs-CTX and FA-FITC-NPs-CTX into tumor cells. HeLa and A549 cells were added into a Petri dish at $1 \times 10^{5}$ cells each and cultured for 24 hours. Then, FITClabeled NPs with the same protein concentration were added and incubated with the cells for 4 hours. Cellular nuclei were stained with DAPI $(1 \mu \mathrm{g} / \mathrm{mL})$.

\section{Hemolysis assay and toxicity evaluation}

Fresh red blood cells (RBCs) were obtained from mouse, centrifuged at $289 \times g$ for 15 minutes at $25^{\circ} \mathrm{C}$, washed with NS three times, and finally diluted to $2 \%$ concentration (v/v) in $1.5 \mathrm{~mL}$ sterile Eppendorf tubes. Tween-CTX, NPs-CTX, and FA-NPs-CTX (CTX concentrations were 5, 10, 20, 50, 100 , and $200 \mu \mathrm{g} / \mathrm{mL}$, respectively) were separately added into $1 \mathrm{~mL}$ of $2 \% \mathrm{RBC}$ standard dispersion, mixed gently, 
and placed in a $37^{\circ} \mathrm{C}$ water bath for 2 hours. NS treatment served as a negative control group, and 1\% Triton X-100 was the positive control. The hemolytic activity was visualized, and then the mixture was centrifuged at $43 \times g$ for 5 minutes. Next, $100 \mu \mathrm{L}$ of the supernatant from each group was added into a 96-well plate and incubated at $37^{\circ} \mathrm{C}$ for 30 minutes. The absorbance of the supernatant was determined at $540 \mathrm{~nm}$. The hemolysis ratio was calculated as follows:

$$
\begin{aligned}
& \text { Hemolysis ratio } \\
& =\frac{\text { Experimental group }- \text { Negative control }}{\text { Positive control }- \text { Negative control }} \times 100 \%
\end{aligned}
$$

Toxicity evaluation was performed for BALB/c mice (male and female). Mice were divided into 15 groups randomly ( $\mathrm{n}=12$, six male and six female). Tween-CTX, NPs-CTX, and FA-NPs-CTX with a series of concentrations of CTX $(0.2,0.6,1.8,5.4$, and $16.2 \mathrm{mg} / \mathrm{kg})$ were injected through the tail vein.

\section{Biodistribution of NPs in nude mice}

In vivo fluorescent images of nude mice were taken using an IVIS in vivo imaging system (Caliper Life Sciences, Hopkinton, MA, USA) with a $745 \mathrm{~nm}$ excitation wavelength and an $800 \mathrm{~nm}$ emission filter. NPs-DiR and FA-NPs-DiR (DiR concentration was $200 \mu \mathrm{g} / \mathrm{mL}$ ) were injected into the tail vein of nude mice. The DiR signals were obtained after $0.5,2$, and 4 hours. After 4 hours, the mice were killed and the tumors, hearts, livers, spleens, lungs, and kidneys were collected for imaging.

\section{In vivo antitumor experiments and histological assessment}

Female nude mice were used for in vivo antitumor experiments. For xenograft generation, $2 \times 10^{7} \mathrm{HeLa}$ cells were implanted subcutaneously into the right hind flank of nude mice. When the average tumor volume increased to $100-150 \mathrm{~mm}^{3}$, the mice were randomly divided into four groups $(\mathrm{n}=5)$. On $0,4,8,12$, and 16 days, CTX, NPs-CTX, and FA-NPs-CTX (equivalent CTX dose of $2 \mathrm{mg} / \mathrm{kg}$ ) were injected through the tail vein. An NS-treated group was used as a negative control. The weight of the nude mice and the length $(a)$ and width $(b)$ of the tumor were measured every 2 days. The tumor volume was calculated as follows:

$$
V=\frac{a \times b \times b}{2}
$$

On the 20th day, the nude mice were euthanized, and the tumors of each group were excised and weighed. Organs including the heart, liver, spleen, lung, and kidney were obtained and fixed in 4\% paraformaldehyde. Histological analysis of these organs was evaluated by H\&E staining.

\section{Statistical analysis}

The data were analyzed using ANOVA, and all graph data are expressed as the mean \pm standard error. Significant differences were calculated using Student's $t$-test, and $P$-values $<0.05$ were considered statistically significant.

\section{Results and discussion Characteristics of NPs-CTX and FA-NPs-CTX}

NPs-CTX were prepared using a self-assembly method, and FA was conjugated to the NPs-CTX through amino groups on HSA. As shown in Table 1, the mean particle size of NPs-CTX was $143 \pm 6 \mathrm{~nm}$, with a polydispersity index (PDI) of $0.132 \pm 0.03$ and a zeta potential of $-21.3 \pm 0.9 \mathrm{mV}$. After FA modification, the particle size of FA-NPs-CTX was $152 \pm 5 \mathrm{~nm}$, which is only slightly larger. The zeta potential of FA-NPs-CTX was reduced to $-26.7 \pm 0.6 \mathrm{mV}$, possibly due to the exchange of positively charged amino groups for the negative charged FA on the NP surface.

As shown in Figure S1, there was an absorption peak at $352 \mathrm{~nm}$ for FA, FA-NHS, and FA-NPs-CTX, which confirms that FA was successfully conjugated to NPs-CTX. Moreover, after trypsin digestion, the content of FA in FA-NPs-CTX was measured using the absorbance at $352 \mathrm{~nm}$. The quantity of FA on the surface of FA-NPs-CTX was $11.96 \pm 3.11 \mu \mathrm{g} / \mathrm{mg}$. Figure 2 shows the SEM images of an NP, indicating a

Table I Physicochemical parameters of NPs-CTX and FA-NPs-CTX

\begin{tabular}{llllll}
\hline Sample & Mean diameter $(\mathbf{n m})$ & Zeta potential $(\mathbf{m V})$ & PDI & EE & EC \\
\hline NPs-CTX & $143 \pm 6$ & $-21.3 \pm 0.9$ & $0.132 \pm 0.03$ & $50.3 \pm 1.8$ & $5.6 \pm 0.4$ \\
FA-NPs-CTX & $152 \pm 5$ & $-26.7 \pm 0.6$ & $0.165 \pm 0.02$ & $50.3 \pm 1.8$ & $5.3 \pm 0.3$ \\
\hline
\end{tabular}

Note: Data are presented as mean \pm SD $(n=3)$.

Abbreviations: EC, encapsulated content; EE, encapsulation efficiency; FA-NPs-CTX, FR-targeted nanoparticles loaded with cabazitaxel; FR, folate receptor; NPs-CTX, cabazitaxel-loaded nanoparticles; PDI, polydispersity index. 

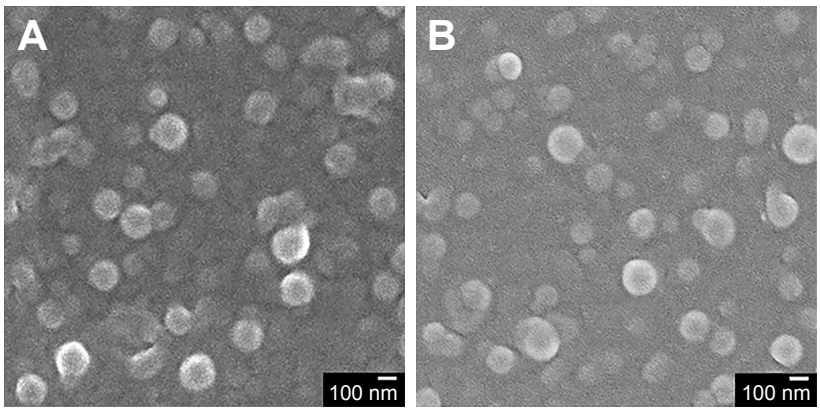

Figure 2 Morphology of (A) NPs-CTX and (B) FA-NPs-CTX imaged by SEM. Note: The scale bar represents $100 \mathrm{~nm}$.

Abbreviations: CTX, cabazitaxel; FA, folic acid; FA-NPs-CTX, FR-targeted nanoparticles loaded with cabazitaxel; FR, folate receptor; NPs-CTX, cabazitaxelloaded nanoparticles; SEM, scanning electron microscopy.

spherical shape. NPs-CTX and FA-NPs-CTX had similar morphology and relatively uniform particle sizes. According to HPLC analysis, $50.3 \% \pm 1.8 \%$ CTX was encapsulated

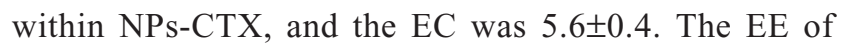
FA-NPs-CTX was the same as that of NPs-CTX because FA-NPs-CTX were prepared from NPs-CTX; however, the EC was slightly decreased to $5.3 \pm 0.3$ due to the loss of CTX during conjugation.

\section{Stability evaluation in vitro}

NPs may interact with proteins in blood circulation and trigger clearance by the reticuloendothelial system (RES). To simulate serum for testing the stability of the NPs (FANPs-CTX and NPs-CTX), 10\% FBS was used. The stability of NPs in NS was also investigated. As shown in Figure S2A, after incubating at $25^{\circ} \mathrm{C}$ and $37^{\circ} \mathrm{C}$ for 48 hours, the particle size increased only slightly in NS, which indicated that both NPs-CTX and NPs-CTX had good stability in NS. However, particle size increased by $40 \mathrm{~nm}$ when the NPs were incubated in 10\% FBS for 8 hours. Nonetheless, FA-NPs-CTX and NPs-CTX were generally stable at $10 \%$ FBS.

The in vitro release of CTX from NPs-CTX and FA-NPsCTX is illustrated in Figure S2B. Upon incubation in PBS containing $0.1 \%$ Tween 80 for 12 hours, $49.9 \% \pm 1.6 \%$ and $48.1 \% \pm 1.4 \%$ of CTX was released from NPs-CTX and FANPs-CTX, respectively. Furthermore, Tween-CTX showed almost total drug release $(86.5 \% \pm 3.0 \%)$. The two NPs exhibited attenuated drug release kinetics, and the modification of the surface of NPs by FA did not affect the release of CTX from FA-NPs-CTX.

\section{Cytotoxicity evaluation in vitro}

The cytotoxicity of FA-NPs-CTX, NPs-CTX, and free CTX was evaluated in HeLa (FR-overexpressing) and A549
(FR-negative) cells using the MTT assay. Three different concentrations of CTX were used to measure cell viability with incubation times of 24,48 , or 72 hours. As shown in Figure 3, FA-NPs-CTX, NPs-CTX, and free CTX all exhibited dose-dependent cytotoxicity. After incubation for 24 hours, cell viability in the presence of free CTX was lower than in the presence of FA-NPs-CTX or NPs-CTX. However, the two NP formulations exhibited enhanced cytotoxicity to A549 cells at longer time points (48 and 72 hours), which suggested that CTX was released from NPs. In HeLa cells, FA-NPs-CTX were more cytotoxic than NPs-CTX at the same concentration of CTX $(P<0.05)$, which could be attributed to FR-mediated uptake. These results suggested that CTX is efficiently released from FA-NPs-CTX and NPs-CTX in both HeLa and A549 cells, and the cytotoxicity of CTX could be enhanced by both nonspecific endocytosis (A549) and FR-mediated endocytosis (HeLa).

\section{Cellular uptake in vitro}

To investigate the efficiency of FA-modified-NP targeting to FR-overexpressing HeLa cells, the uptake of FITC-labeled NPs (FA-NPs-CTX and FA-FITC-NPs-CTX) was analyzed by flow cytometry. FR-negative A549 cells were used as negative control. As shown in Figure 4, the cellular uptake increased over time both in HeLa and A549 cells. The fluorescence intensity of HeLa cells treated with FA-FITCNPs-CTX was much stronger than that of cells treated with FITC-NPs-CTX. The cellular uptake of FA-FITC-NPs-CTX was 1.87 times that of FITC-NPs-CTX at 4 hours. In contrast, there was no significant difference in the uptake of the two formulations in A549 cells. To further verify FA-mediated endocytosis, FR was blocked with excess FA prior to the addition of FA-FITC-NPs-CTX (FA + FA-FITC-NPs-CTX) to HeLa cells. As shown in Figure 4A, the cellular uptake was blocked, compared with the result from FA-FITC-NPs-CTX addition in the absence of FA, suggesting that uptake was through FR-mediated endocytosis.

Cellular uptake was visualized using LCSM. As shown in Figure 5A, the fluorescence intensity of FA-FITC-NPs-CTXtreated HeLa cells was significantly stronger than that of cells treated with FITC-NPs-CTX or FA + FA-FITC-NPs-CTX. This result demonstrated that the uptake of FA-FITC-NPsCTX by HeLa cells was enhanced by FR-mediated endocytosis. In A549 cells, there were no significant differences in fluorescence intensity between cells treated with the two formulations (Figure 5B). As observed from the qualitative and quantitative cellular uptake results, FA-modified NPs show enhanced uptake by HeLa cells via FR-mediated endocytosis. 


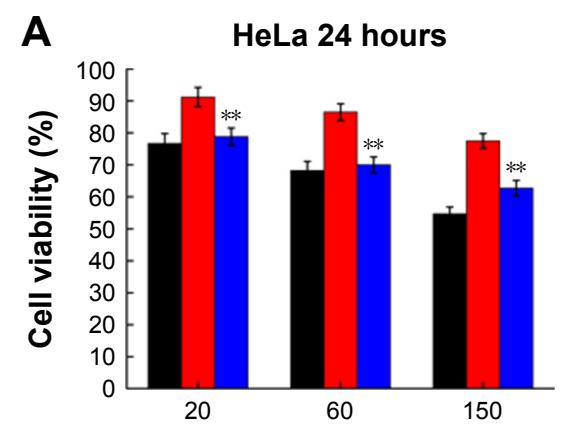

Concentration $(\mu \mathrm{g} / \mathrm{mL})$

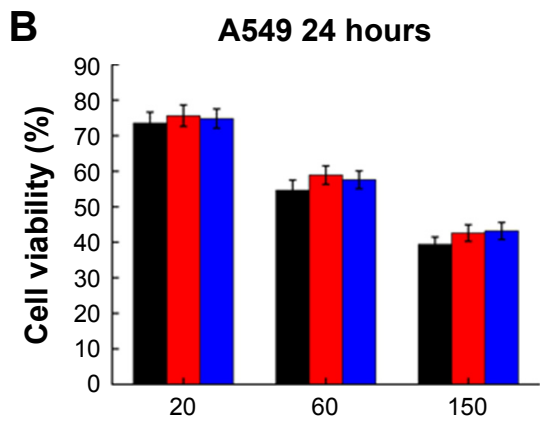

Concentration $(\mu \mathrm{g} / \mathrm{mL})$

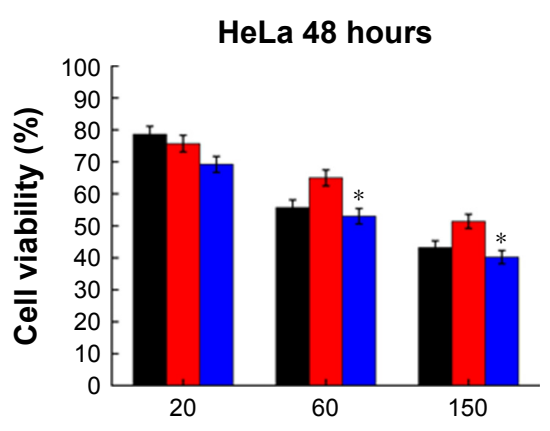

Concentration $(\mu \mathrm{g} / \mathrm{mL})$

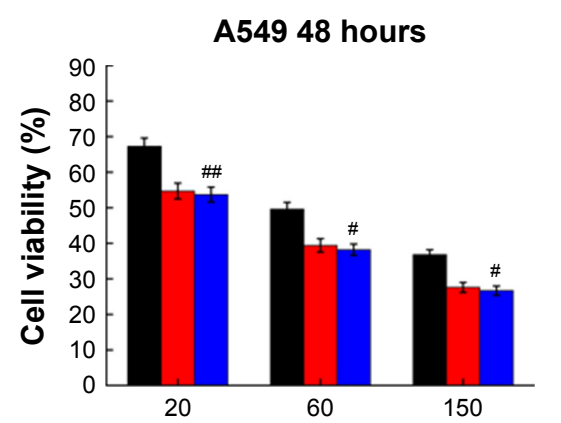

Concentration $(\mu \mathrm{g} / \mathrm{mL})$

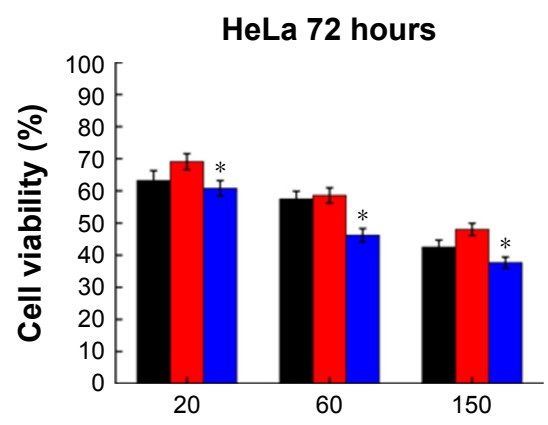

Concentration $(\mu \mathrm{g} / \mathrm{mL})$

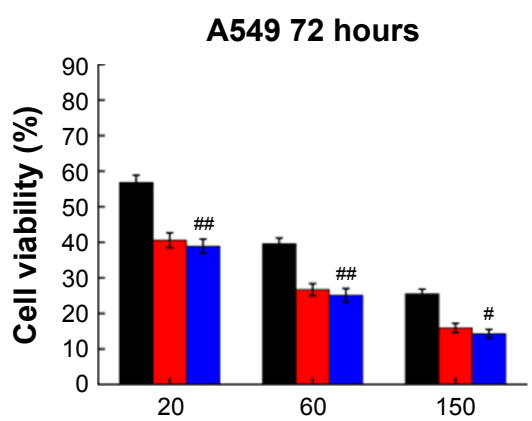

Concentration $(\mu \mathrm{g} / \mathrm{mL})$

\section{Free CTX $\square$ NPs-CTX $\square$ FA-NPs-CTX}

Figure 3 Cytotoxicity of free CTX, NPs-CTX, and FA-NPs-CTX.

Notes: (A) HeLa and (B) A549 cells were treated for 24,48 , and 72 hours with $C T X$ formulations. Data are presented as the mean \pm SD $(n=6)$. $* P<0.05$ and $* * P<0.01$ vs NPs-CTX; ${ }^{*} P<0.05$ and ${ }^{\# \#} P<0.01$ vs free CTX.

Abbreviations: CTX, cabazitaxel; FA, folic acid; FA-NPs-CTX, FR-targeted nanoparticles loaded with cabazitaxel; FR, folate receptor; Free CTX, cabazitaxel dissolved in ethanol; NPs-CTX, cabazitaxel-loaded nanoparticles.

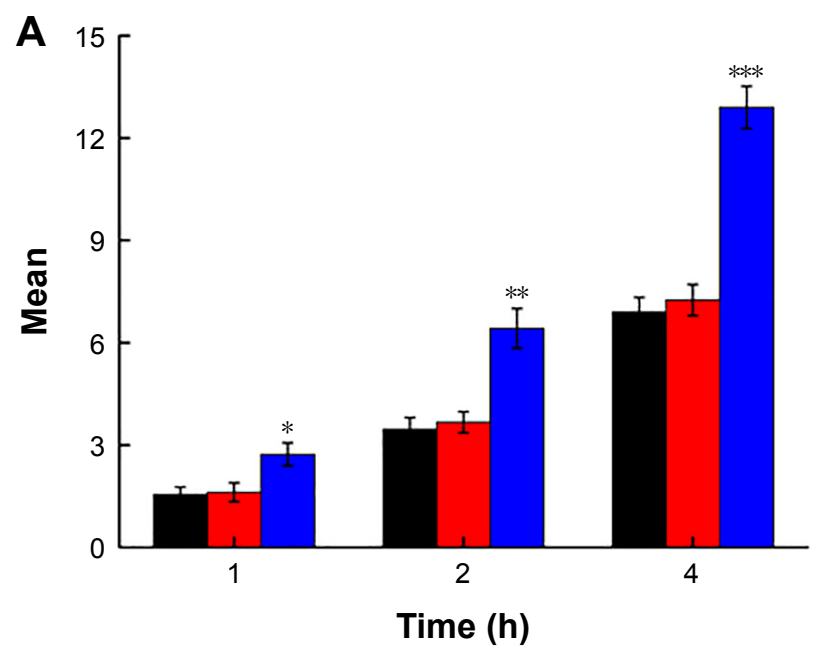

Time (h)

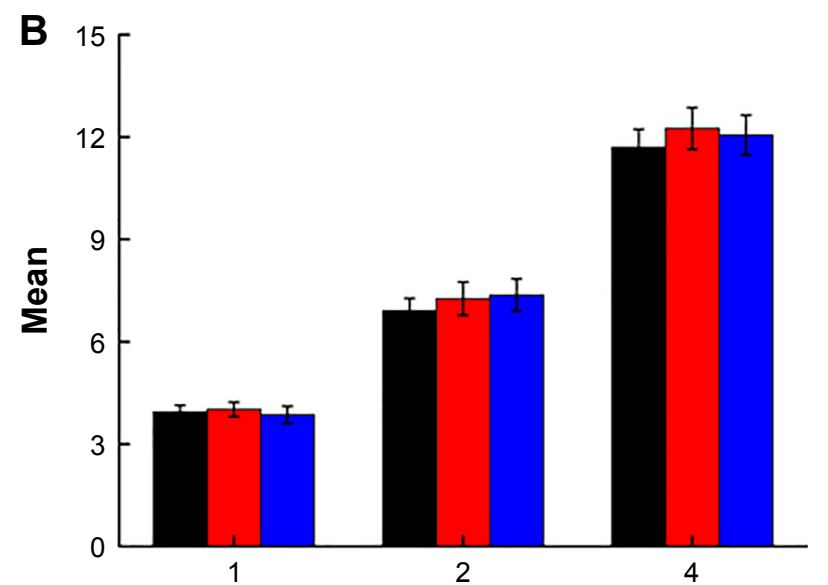

Time (h)

FITC-NPS-CTX

FA + FA-FITC-NPs-CTX

FA-FITC-NPs-CTX

Figure 4 Quantitative analyses of uptake of FITC-labeled NPs at I, 2, and 4 hours by flow cytometry.

Notes: (A) Florescence intensity of HeLa cells treated with FITC-NPs-CTX, FA-FITC-NPs-CTX, and FA + FA-FITC-NPs-CTX for I, 2, and 4 hours. (B) Florescence intensity of $A 549$ cells treated with FITC-NPs-CTX, FA-FITC-NPs-CTX, and FA + FA-FITC-NPs-CTX for I, 2, and 4 hours. Data are presented as the means \pm SD ( $=3$ ). $* P<0.05$, $* * P<0.01$, and $* * * P<0.001$ vs NPs-CTX.

Abbreviations: CTX, cabazitaxel; FA, folic acid; FA + FA-FITC-NPs-CTX, free FA was added prior to the addition of FA-FITC-NPs-CTX; FA-FITC-NPs-CTX, FR-targeted nanoparticles loaded with cabazitaxel and labeled with FITC; FITC, fluorescein isothiocyanate; FITC-NPs-CTX, cabazitaxel-loaded nanoparticles labeled with FITC; FR, folate receptor. 
A

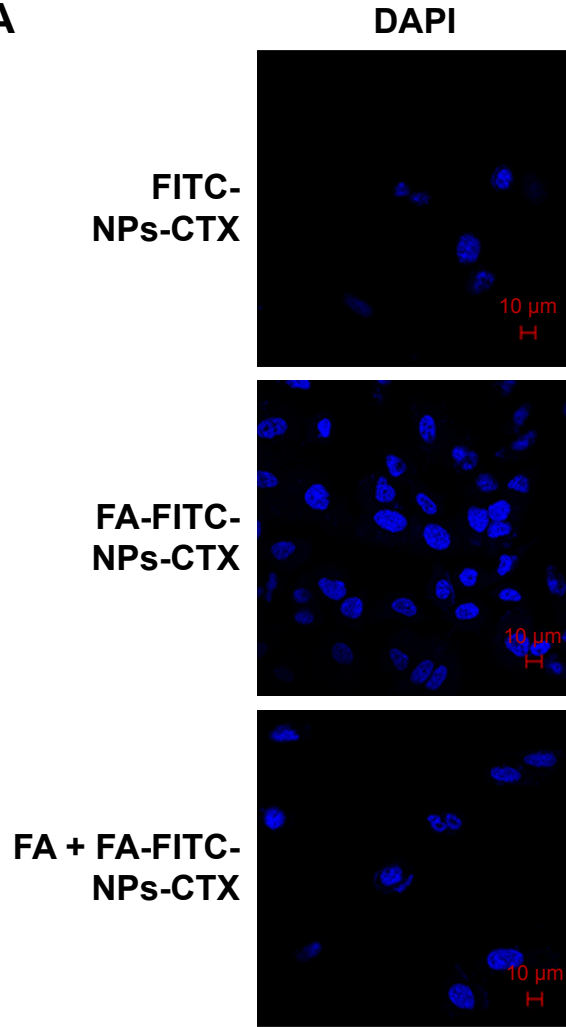

B

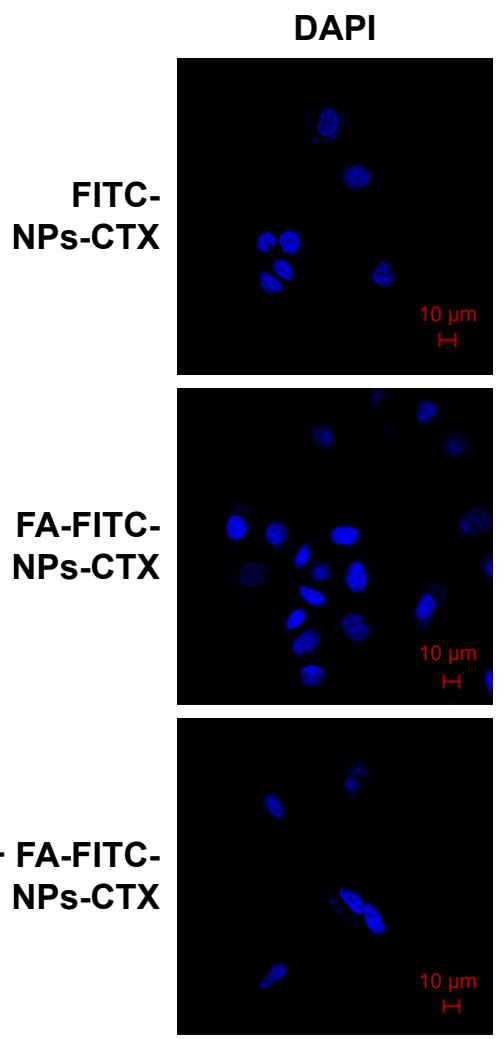

FITC
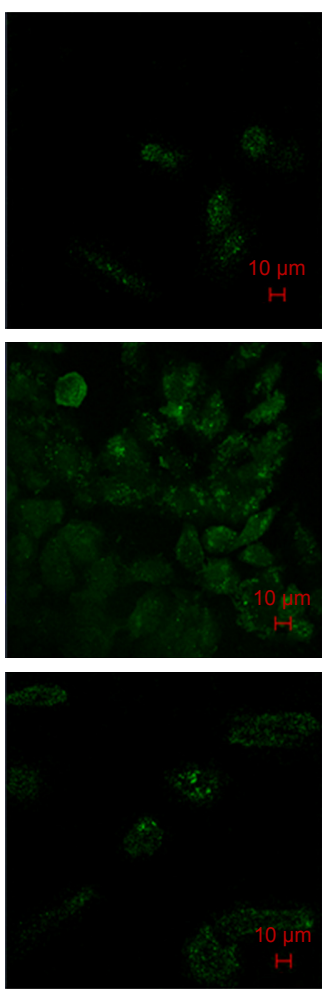

FITC
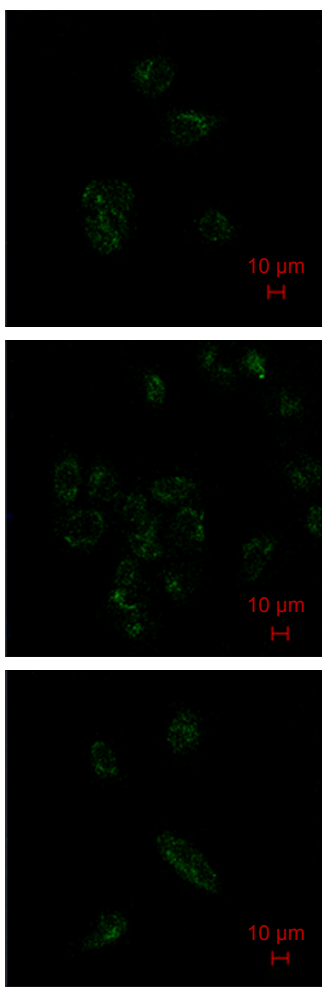

DIC
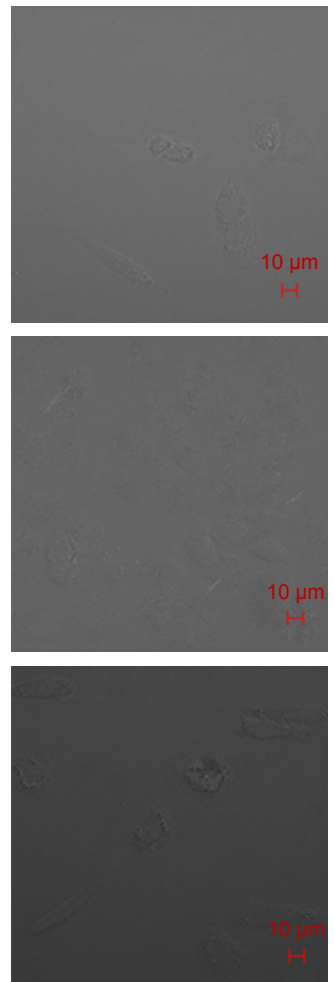

DIC
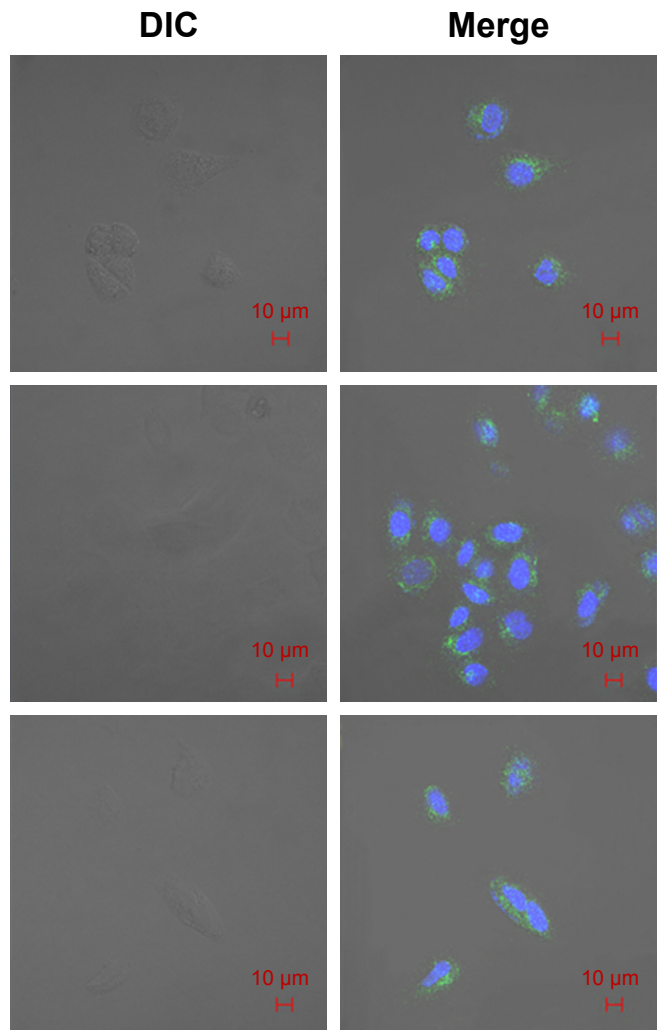

Figure 5 CLSM images of (A) HeLa and (B) A549 cells after incubation with FITC-NPs-CTX, FA-FITC-NPs-CTX, or FA + FA-FITC-NPs-CTX for 4 hours.

Abbreviations: CLSM, confocal laser scanning microscopy; CTX, cabazitaxel; DIC, differential interference contrast; FA, folic acid; FA + FA-FITC-NPs-CTX, free FA was added prior to the addition of FA-FITC-NPs-CTX; FA-FITC-NPs-CTX, FR-targeted nanoparticles loaded with cabazitaxel and labeled with FITC; FITC, fluorescein isothiocyanate; FITC-NPs-CTX, cabazitaxel-loaded nanoparticles labeled with FITC; FR, folate receptor. 


\section{Hemolysis assay and toxicity evaluation}

Hemolysis experiments were used to test the effects of FANPs-CTX, NPs-CTX, and Tween-CTX on RBCs. As shown in Figure 6A and B, Tween-CTX exhibited strong hemolytic effects compared with the FA-NPs-CTX and NPs-CTX formulations. According to Table 2, hemolysis occurred in $60.43 \% \pm 0.91 \%$ of RBCs in the presence of $5 \mu \mathrm{g} / \mathrm{mL}$ TweenCTX, whereas only $16.13 \% \pm 0.15 \%$ and $16.74 \% \pm 0.15 \%$ hemolysis occurred with $200 \mu \mathrm{g} / \mathrm{mL}$ NPs-CTX and FA-NPs-
CTX, respectively. These results demonstrated that NPs-CTX and FA-NPs-CTX have enhanced hemocompatibility and that FA modification affects hemocompatibility. Furthermore, Figure $6 \mathrm{C}$ and D showed that the median lethal dose (LD50) of Tween-CTX in mice was $5.68 \mathrm{mg} / \mathrm{kg}$, whereas there was no death in the FA-NPs-CTX and NPs-CTX groups at drug concentrations up to $400 \mathrm{mg} / \mathrm{kg}$. These results indicate that the safety of CTX had been greatly improved (at least 70-fold) due to the encapsulation of NPs and FA-NPs.

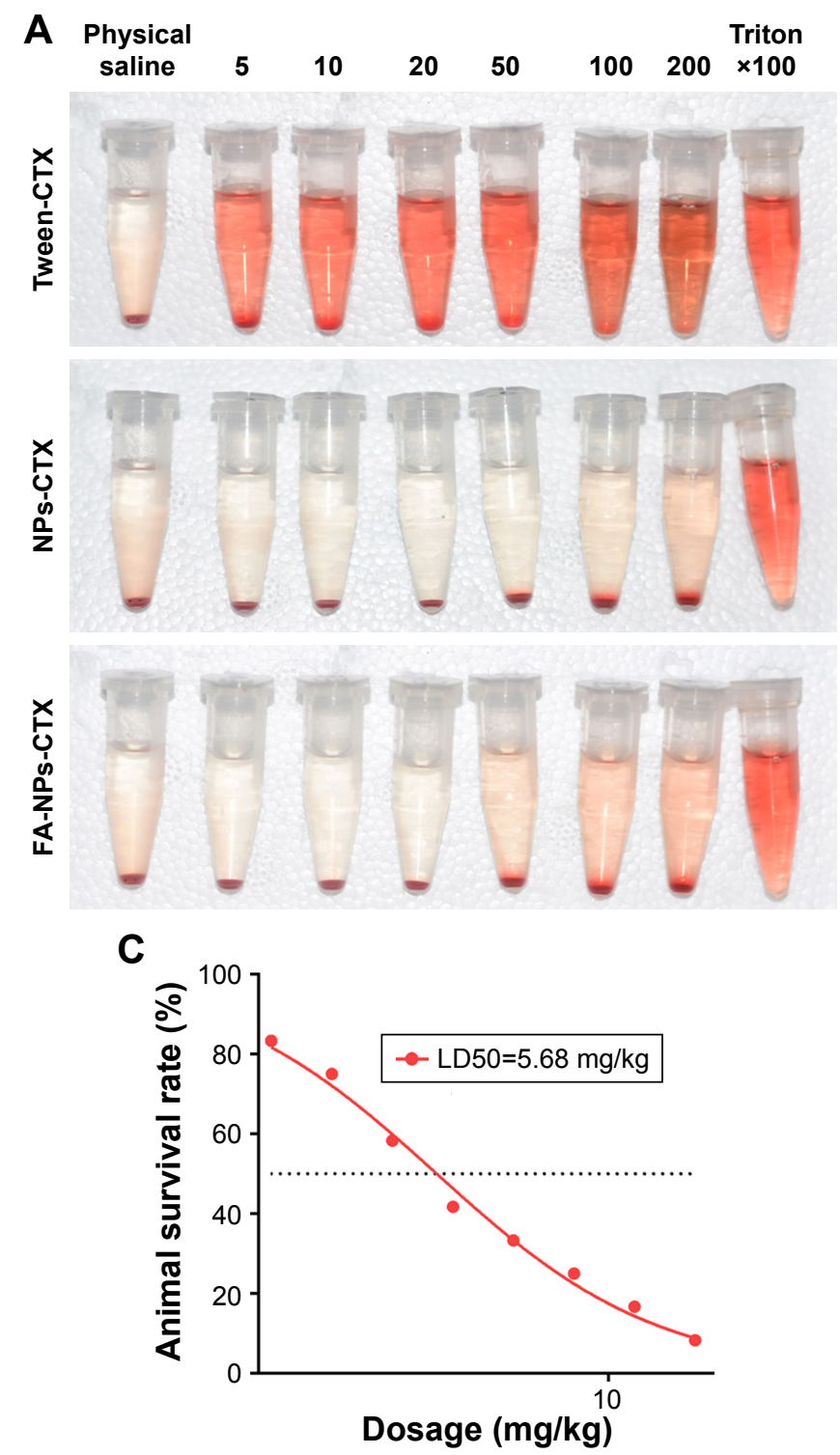

B
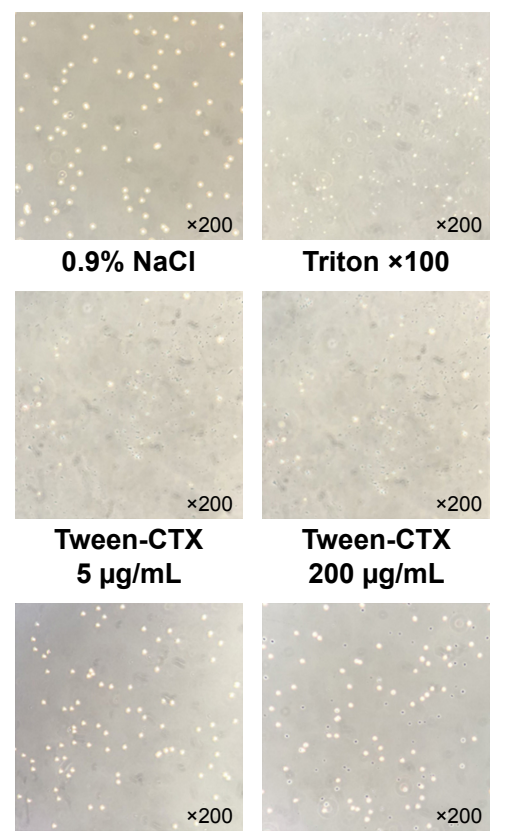

$200 \mu \mathrm{g} / \mathrm{mL}$

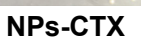

$5 \mu \mathrm{g} / \mathrm{mL}$
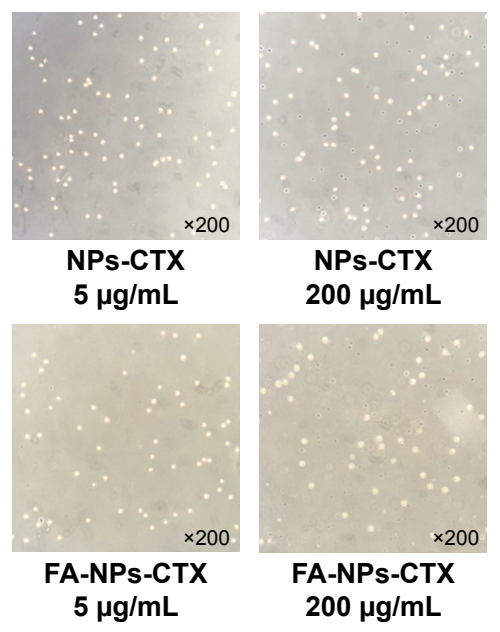

D

\begin{tabular}{ll}
\hline Sample & LD50 $(\mathbf{m g} / \mathbf{k g})$ \\
\hline Tween-CTX & 5.68 \\
NPs-CTX & $>400$ \\
FA-NPs-CTX & $>400$ \\
\hline
\end{tabular}

Figure 6 Hemolysis and toxicity evaluation of three formulations.

Notes: (A) Photograph of hemolysis of red blood cells treated with Tween-CTX, NPs-CTX, and FA-NPs-CTX. (B) Optical image of hemolytic study of Tween-CTX, NPs-CTX, and FA-NPs-CTX. (C) LD50 evaluation of Tween-CTX. (D) LD50 values of Tween-CTX, NPs-CTX, and FA-NPs-CTX.

Abbreviations: CTX, cabazitaxel; FA, folic acid; FA-NPs-CTX, FR-targeted nanoparticles loaded with cabazitaxel; FR, folate receptor; LD50, median lethal dose; NPs-CTX, cabazitaxel-loaded nanoparticles; Tween-CTX, CTX dissolved in a mixture of Tween 80 and I3\% ethanol ( $w / w)$ at a ratio of I:4 (v/v). 
Table 2 Hemolysis ratio obtained from microplate reader at $540 \mathrm{~nm}$

\begin{tabular}{lllllll}
\hline & $\mathbf{5}$ & $\mathbf{1 0}$ & $\mathbf{2 5}$ & $\mathbf{5 0}$ & $\mathbf{1 0 0}$ & $\mathbf{2 0 0}$ \\
\hline NPs-CTX & $0.51 \pm 0.15$ & $0.60 \pm 0.15$ & $0.66 \pm 0.10$ & $2.13 \pm 0.15$ & $6.85 \pm 0.30$ & $16.13 \pm 0.15$ \\
FA-NPs-CTX & $0.46 \pm 0.09$ & $0.61 \pm 0.15$ & $0.68 \pm 0.12$ & $2.74 \pm 0.15$ & $10.35 \pm 0.15$ & $16.74 \pm 0.15$ \\
Tween-CTX & $60.43 \pm 0.91$ & $73.36 \pm 0.76$ & $77.63 \pm 1.07$ & $84.02 \pm 0.46$ & $89.9 \pm 0.38$ & $95.9 \pm 0.67$ \\
\hline
\end{tabular}

Note: Data are presented as mean \pm SD $(n=3)$.

Abbreviations: FA-NPs-CTX, FR-targeted nanoparticles loaded with cabazitaxel; FR, folate receptor; NPs-CTX, cabazitaxel-loaded nanoparticles; Tween-CTX, CTX dissolved in a mixture of Tween 80 and $13 \%$ ethanol $(\mathrm{w} / \mathrm{w})$ at a ratio of $1: 4(\mathrm{v} / \mathrm{v})$.

\section{Antitumor activity in vivo}

To investigate whether FA-targeted NPs (FA-NPs-CTX) can accumulate at the tumor site in nude mice via FR, FA-NPsDiR and NPs-DiR with the same DiR content were injected into nude mice via the tail vein. Because DiR was encapsulated into HSA NPs instead of CTX, the intensity of DiR represented the distribution of the drug in the body. As shown in Figure 7A, DiR fluorescence was observed 0.5 hours after injection, and the intensity increased over time. Compared to NPs-DiR, FA-NPs-DiR showed stronger fluorescence at the tumor site, which indicated enhanced delivery by FR-meditated endocytosis. Figure 7B illustrates the distribution of DiR in different organs. For NPs-DiR, there was some accumulation of fluorescence at the tumor site, which might be attributed to the EPR effect. Even though some fluorescence was distributed in the liver, lung, and kidney, the
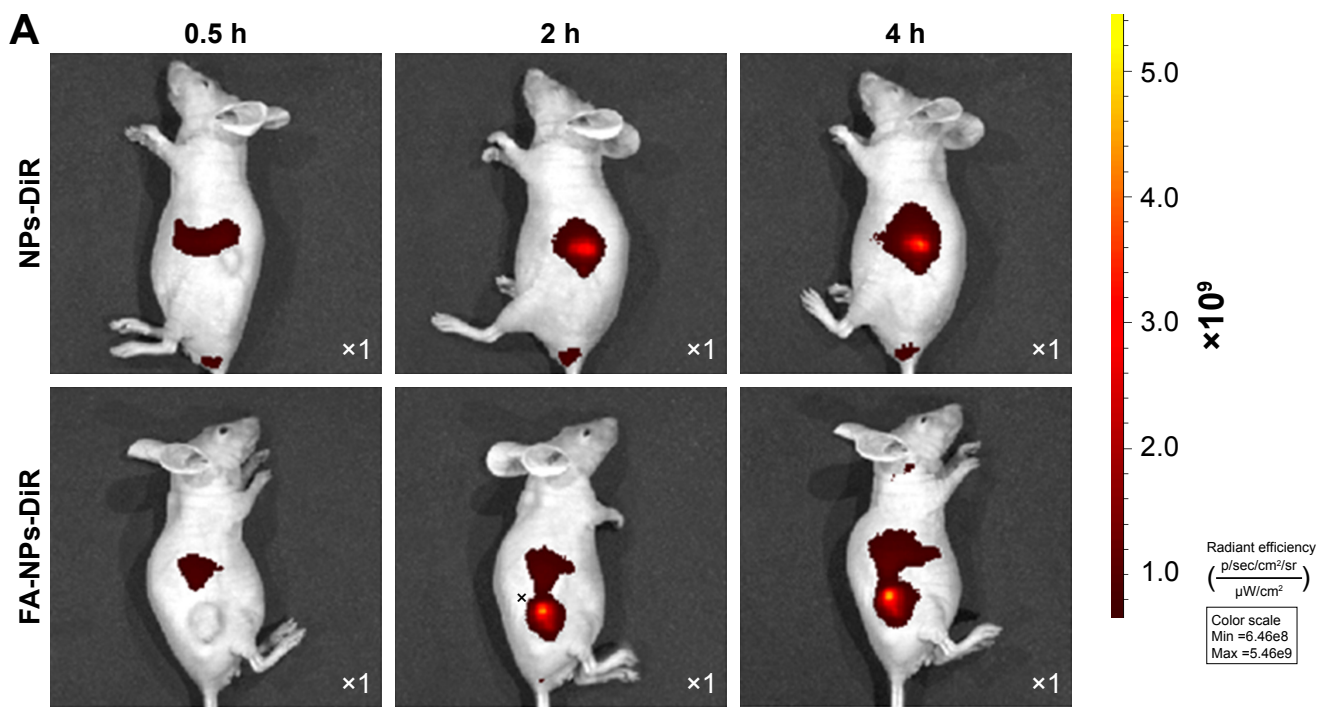

B
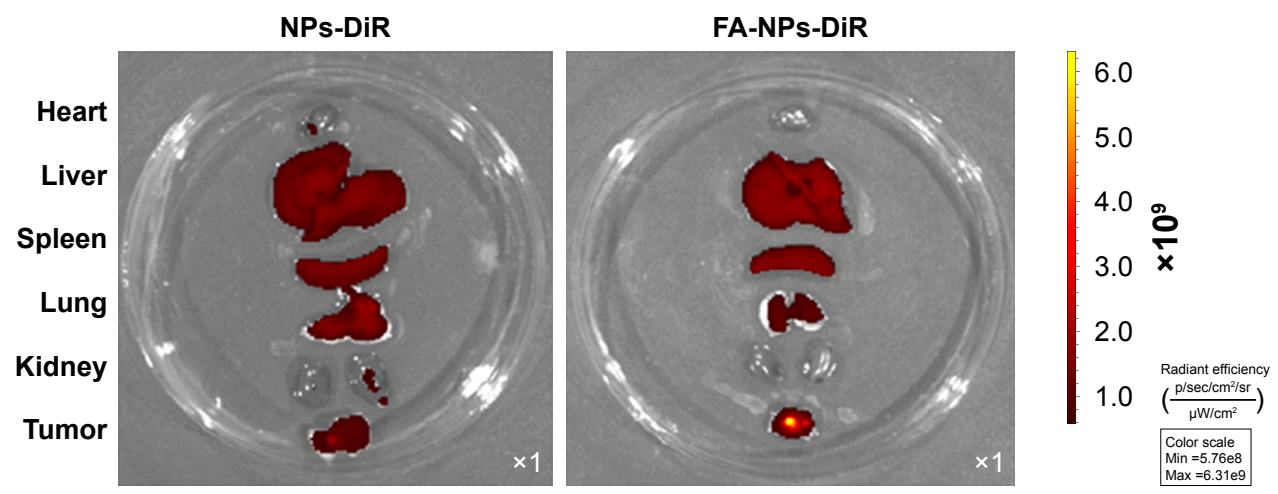

Figure 7 In vivo distribution and ex vivo imaging of organs and tumors in HeLa xenograft-bearing nude mice.

Notes: (A) Fluorescence images of DiR obtained 0.5, 2, and 4 hours after the intravenous injection of NPs-DiR and FA-NPs-DiR. (B) Ex vivo fluorescence images of organs and tumors obtained 4 hours after injection of NPs-DiR and FA-NPs-DiR; for each petri dish, from top to bottom, the organs were the heart, liver, spleen, lung, kidney, and tumor.

Abbreviations: DiR, I,I'-dioctadecyl-3,3,3',3'-tetramethyl indotricarbocyanine; FA, folic acid; FA-NPs-DiR, FR-targeted nanoparticles loaded with DiR; FR, folate receptor; NPs-DiR, DiR-loaded nanoparticles. 
fluorescence intensity of FA-NPs-DiR in tumors was much higher than that seen in mice treated with NPs-DiR. These results demonstrated that through the targeting effect of FA, FA-NPs-DiR can be efficiently accumulated in tumor sites.

To determine the efficacy of tumor growth inhibition by FA-NPs-CTX, a HeLa xenograft model was adopted. As shown in Figure 8A, C, and D, all three formulations (FA-NPs-CTX, NPs-CTX, and Tween-CTX) inhibited tumor growth $(* * P<0.01)$; Tween-CTX showed the greatest inhibition, whereas tumors treated with NS (Control) showed no tumor suppression after 20 days. Specifically, the tumor volume of FA-NPs-CTX increased from $158.5 \pm 17.5 \mathrm{~mm}^{3}$ to $213.6 \pm 96.5 \mathrm{~mm}^{3}$, whereas tumors expanded from $158.4 \pm 16.9 \mathrm{~mm}^{3}$ to $374.7 \pm 123.1 \mathrm{~mm}^{3}$ in NPs-CTX-treated mice, which suggests that FA-NPs-CTX have greater tumor inhibitory capacity $\left({ }^{\#} P<0.05\right)$. The body weights of the mice in the different groups were recorded along with the tumor volumes. As shown in Figure 8B, the body weight of nude mice treated with Tween-CTX decreased more rapidly than the body weight in the other three groups, which demonstrated the severe side effects of Tween-CTX; this was confirmed by H\&E staining. As shown in Figure 9, there was significant tissue damage in the heart, liver, lung, and kidney in the Tween-CTX group, whereas only slight
A

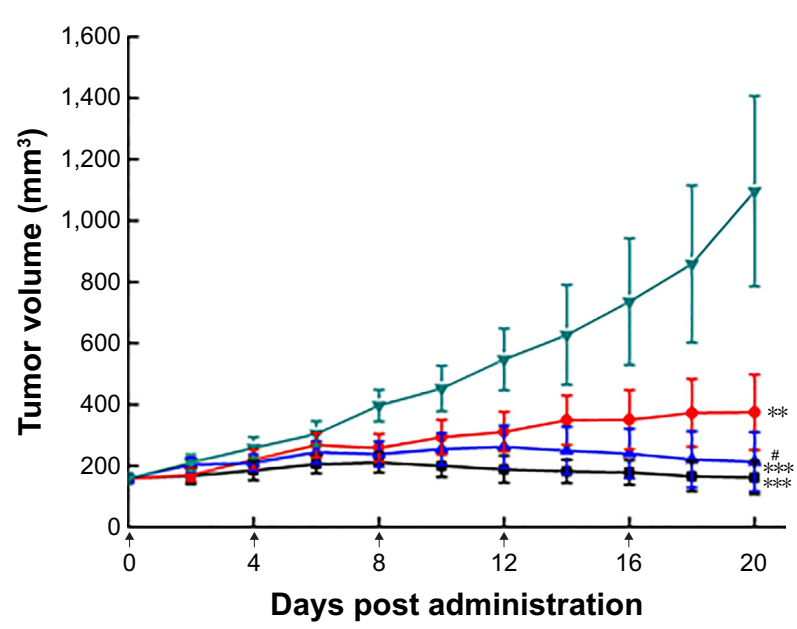

B

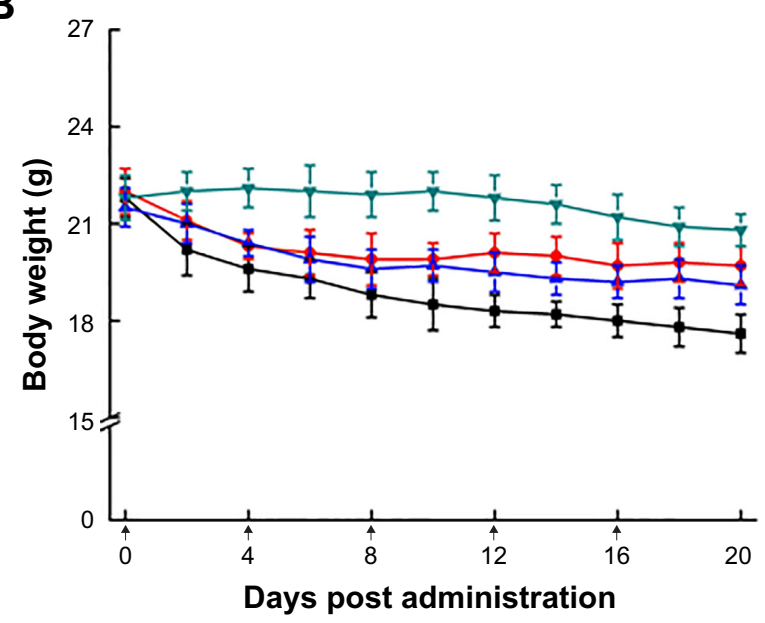

C

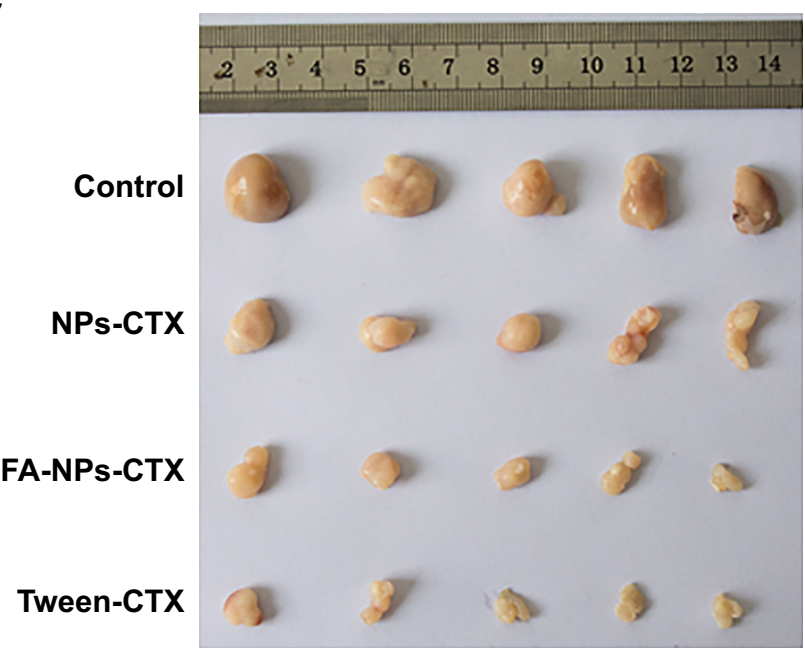

D

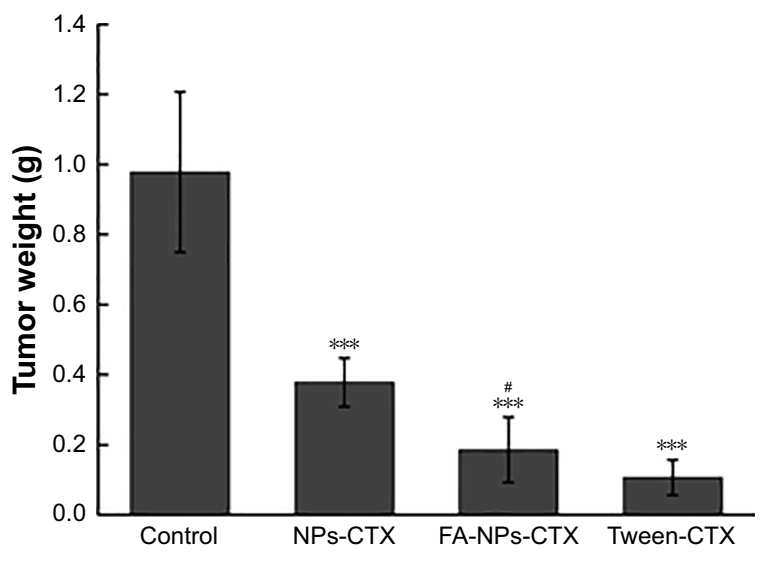

Figure 8 In vivo antitumor evaluation of different formulations against HeLa xenografts in nude mice after the intravenous injection of NS (control), Tween-CTX, NPs-CTX, and FA-NPs-CTX.

Notes: (A) Change of tumor volume. (B) Change of body weight. (C) Image of tumors obtained from nude mice at Day 20. (D) Tumor weight at Day 20 after excision from mice. Data are presented as the mean \pm SD $(n=5)$. $* * p<0.01$, *** $P<0.00$ I vs control; ${ }^{*} P<0.05$ vs NPs-CTX.

Abbreviations: CTX, cabazitaxel; FA, folic acid; FA-NPs-CTX, FR-targeted nanoparticles loaded with cabazitaxel; FR, folate receptor; NS, normal saline; NPs-CTX, cabazitaxel-loaded nanoparticles; Tween-CTX, CTX dissolved in a mixture of Tween 80 and I3\% ethanol ( $w / w)$ at a ratio of I:4 (v/v). 

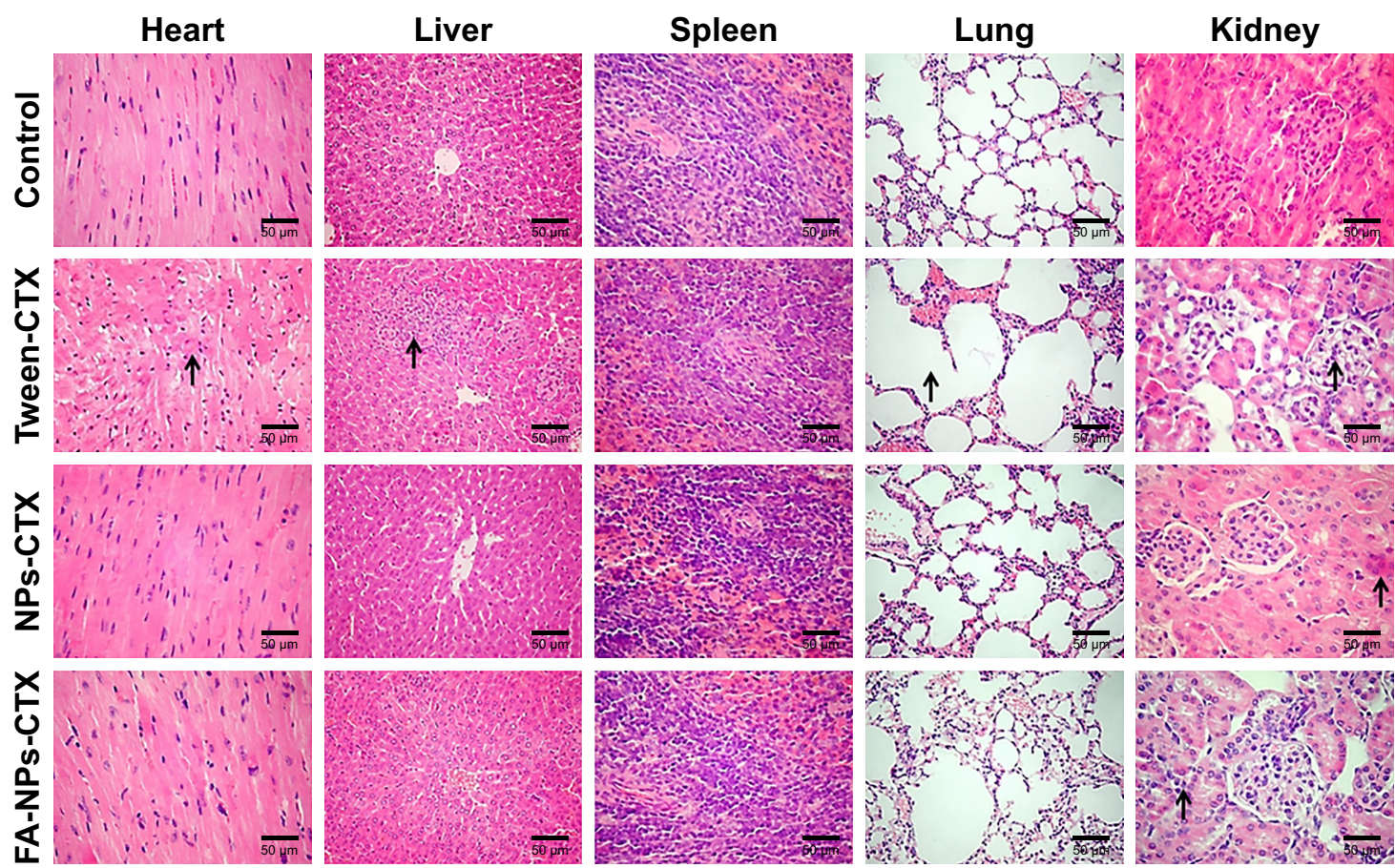

Figure 9 Histological images of major organs excised from HeLa tumor-bearing nude mice after treatment with NS (control), Tween-CTX, NPs-CTX, and FA-NPs-CTX at Day 20.

Note: The images were obtained using a digital microscope at 400× magnification, and major tissue damages are marked with arrows.

Abbreviations: CTX, cabazitaxel; FA, folic acid; FA-NPs-CTX, FR-targeted nanoparticles loaded with cabazitaxel; NPs-CTX, cabazitaxel-loaded nanoparticles; NS, normal saline; Tween-CTX, CTX dissolved in a mixture of Tween 80 and I3\% ethanol $(\mathrm{w} / \mathrm{w})$ at a ratio of I:4 (v/v).

damage to the kidney was observed in the FA-NPs-CTX and NPs-CTX groups. In conclusion, therapeutic efficacy and safety of CTX were greatly improved after loading into NPs, especially FA-NPs.

\section{Conclusion}

In a previous article, we showed that NPs-CTX are highly effective in prostate tumor therapy. Here, after modification with FA, FA-NPs-CTX showed good biocompatibility and physicochemical properties, such as colloidal stability and sustained release. Moreover, our studies demonstrate that FA-NPs-CTX significantly increase cellular uptake efficiency through FR-mediated endocytosis in HeLa cells and enhanced cell growth inhibition. Furthermore, the accumulation of FA-NPs-CTX at the tumor site was also significantly increased. Importantly, FA-NPs-CTX exhibited remarkable tumor inhibition while reducing side effects such as toxicity. In summary, the FA-NPs studied here have great potential for the targeted delivery of drugs and warrant further investigation.

\section{Acknowledgment}

This research was supported by grants from the National Natural Science Foundation of China (number 81502999), the Jilin Province Science and Technology Development
Program (number 20170414039 GH), the Jilin Province Industrial Innovation Project (number 2017C026), and the Special Financial Grant from the China Postdoctoral Science Foundation (number 2017T100374).

\section{Disclosure}

The authors report no conflicts of interest in this work.

\section{References}

1. Abidi A. Cabazitaxel: A novel taxane for metastatic castration-resistant prostate cancer-current implications and future prospects. $J$ Pharmacol Pharmacother. 2013;4(4):230-237.

2. Jarvis C, Nelius T, Martinez-Marin D, Sennoune SR, Filleur S. Cabazitaxel regimens inhibit the growth of prostate cancer cells and enhances the anti-tumor properties of PEDF with various efficacy and toxicity. Prostate. 2018;78(12):905-914.

3. Hoang B, Ernsting MJ, Tang WS, et al. Cabazitaxel-conjugated nanoparticles for docetaxel-resistant and bone metastatic prostate cancer. Cancer Lett. 2017;410:169-179.

4. Hongo H, Kosaka T, Oya M. Analysis of cabazitaxel-resistant mechanism in human castration-resistant prostate cancer. Cancer Sci. 2018; 109(9):2937-2945.

5. Ojima I, Lichtenthal B, Lee S, Wang C, Wang X. Taxane anticancer agents: a patent perspective. Expert Opin Ther Pat. 2016;26(1):1-20.

6. Zhuang B, du L, Xu H, et al. Self-assembled micelle loading cabazitaxel for therapy of lung cancer. Int J Pharm. 2016;499(1-2):146-155.

7. Coors EA, Seybold H, Merk HF, Mahler V. Polysorbate 80 in medical products and nonimmunologic anaphylactoid reactions. Ann Allergy Asthma Immunol. 2005;95(6):593-599.

8. Kratz F. Albumin as a drug carrier: design of prodrugs, drug conjugates and nanoparticles. J Control Release. 2008;132(3):171-183. 
9. Miele E, Spinelli GP, Miele E, Tomao F, Tomao S. Albumin-bound formulation of paclitaxel (Abraxane ABI-007) in the treatment of breast cancer. Int J Nanomedicine. 2009;4:99-105.

10. Liu Z, Chen X. Simple bioconjugate chemistry serves great clinical advances: albumin as a versatile platform for diagnosis and precision therapy. Chem Soc Rev. 2016;45(5):1432-1456.

11. Chen Q, Wang X, Wang C, Feng L, Li Y, Liu Z. Drug-induced selfassembly of modified albumins as nano-theranostics for tumor-targeted combination therapy. ACS Nano. 2015;9(5):5223-5233.

12. Kinoshita R, Ishima $Y$, Chuang VTG, et al. Improved anticancer effects of albumin-bound paclitaxel nanoparticle via augmentation of EPR effect and albumin-protein interactions using S-nitrosated human serum albumin dimer. Biomaterials. 2017;140:162-169.

13. Chen Q, Liang C, Wang X, He J, Li Y, Liu Z. An albumin-based theranostic nano-agent for dual-modal imaging guided photothermal therapy to inhibit lymphatic metastasis of cancer post surgery. Biomaterials. 2014;35(34):9355-9362.

14. Lu L, Ding Y, Zhang Y, et al. Antibody-modified liposomes for tumortargeting delivery of timosaponin AIII. Int J Nanomedicine. 2018;13: 1927-1944.

15. Chen C, Ke J, Zhou XE, et al. Structural basis for molecular recognition of folic acid by folate receptors. Nature. 2013;500(7463):486-489.
16. Bahrami B, Mohammadnia-Afrouzi M, Bakhshaei P, et al. Folateconjugated nanoparticles as a potent therapeutic approach in targeted cancer therapy. Tumour Biol. 2015;36(8):5727-5742.

17. Wang X, Li J, Wang Y, et al. A folate receptor-targeting nanoparticle minimizes drug resistance in a human cancer model. ACS Nano. 2011;5(8) 6184-6194.

18. Werner ME, Copp JA, Karve S, et al. Folate-targeted polymeric nanoparticle formulation of docetaxel is an effective molecularly targeted radiosensitizer with efficacy dependent on the timing of radiotherapy. ACS Nano. 2011;5(11):8990-8998.

19. Sun L, Wei Z, Chen H, et al. Folic acid-functionalized up-conversion nanoparticles: toxicity studies in vivo and in vitro and targeted imaging applications. Nanoscale. 2014;6(15):8878-8883.

20. Qu N, Lee RJ, Sun Y, et al. Cabazitaxel-loaded human serum albumin nanoparticles as a therapeutic agent against prostate cancer. Int $J$ Nanomedicine. 2016;11:3451-3459.

21. Qu N, Sun Y, Xie J, Teng L. Preparation and Evaluation of in vitro Self-assembling HSA Nanoparticles for Cabazitaxel. Anticancer Agents Med Chem. 2017;17(2):294-300.

22. Dubey RD, Alam N, Saneja A, et al. Development and evaluation of folate functionalized albumin nanoparticles for targeted delivery of gemcitabine. Int J Pharm. 2015;492(1-2):80-91. 


\section{Supplementary materials}

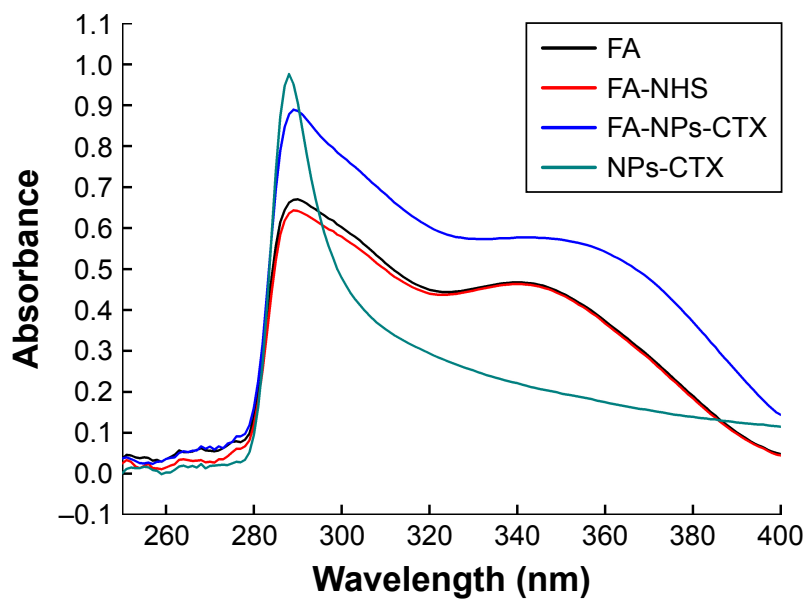

Figure SI Spectral scanning by UV spectrophotometry for determining the content of FA in FA-NPs-CTX.

Abbreviations: FA, folic acid; FA-NHS, folic acid N-hydroxysuccinimide ester; NPs-CTX, cabazitaxel-loaded nanoparticles; FA-NPs-CTX, FR-targeted nanoparticles loaded with cabazitaxel; FR, folate receptor.
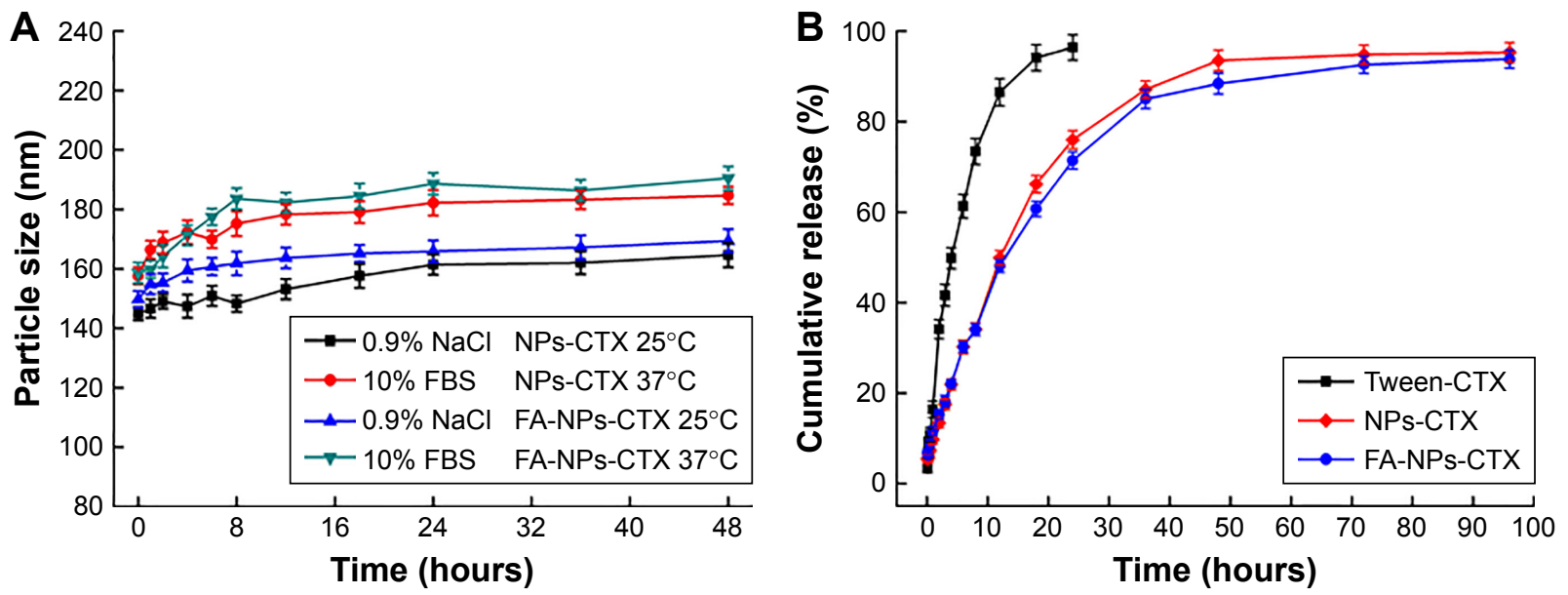

Figure S2 Characterization of NPs-CTX and FA-NPs-CTX.

Notes: (A) Stability of NPs-CTX and FA-NPs-CTX in NS and $10 \%$ FBS at $25^{\circ} \mathrm{C}$ and $37^{\circ} \mathrm{C}$, respectively. (B) Drug release from Tween-CTX, NPs-CTX, and FA-NPs-CTX in PBS containing 0.1\% Tween 80. $n=3$.

Abbreviations: FA, folic acid; FA-NPs-CTX, FR-targeted nanoparticles loaded with cabazitaxel; FR, folate receptor; NPs-CTX, cabazitaxel-loaded nanoparticles; Tween-CTX, CTX dissolved in a mixture of Tween 80 and $13 \%$ ethanol $(\mathrm{w} / \mathrm{w})$ and the ratio was I:4 (v/v); NS, normal saline.

\section{Publish your work in this journal}

The International Journal of Nanomedicine is an international, peerreviewed journal focusing on the application of nanotechnology in diagnostics, therapeutics, and drug delivery systems throughout the biomedical field. This journal is indexed on PubMed Central, MedLine, CAS, SciSearch ${ }^{\circledR}$, Current Contents $® /$ Clinical Medicine,
Journal Citation Reports/Science Edition, EMBase, Scopus and the Elsevier Bibliographic databases. The manuscript management system is completely online and includes a very quick and fair peer-review system, which is all easy to use. Visit http://www.dovepress.com/ testimonials.php to read real quotes from published authors. 